\title{
Structure and Evolution of the Mitochondrial DNA Complete Control Region in the Lizard Lacerta dugesii (Lacertidae, Sauria)
}

\author{
António Brehm, ${ }^{1}$ D. James Harris, ${ }^{2}$ Cíntia Alves, ${ }^{3}$ José Jesus, ${ }^{1}$ Fabienne Thomarat, ${ }^{4}$ Luís Vicente ${ }^{5}$ \\ ${ }^{1}$ Center of Macaronesian Studies, University of Madeira, Campus da Penteada, 9000 Funchal, Portugal \\ ${ }^{2}$ Centro de Estudos de Ciência Animal (CECA), ICETA-UP, Campus Agrario de Vairão, 4485-661 Vila do Conde, Portugal \\ ${ }^{3}$ IPATIMUP, University of Porto, Rua Dr. Roberto Frias, 4000 Porto, Portugal \\ ${ }^{4}$ Laboratoire de Biométrie et Biologie Evolutive, UMR CNRS 5558, Université Lyon I, 69622 Villeurbanne, France \\ ${ }^{5}$ Centre of Environmental Biology, Department of Zoology and Anthropology, Science, Faculty of Lisbon, University of Lisbon, \\ C2 Campo Grande, 1700 Lisbon, Portugal
}

Received: 12 December 2001 / Accepted: 11 July 2002

\begin{abstract}
We sequenced the complete control region (CR) and adjacent tRNAs, partial 12S rRNA, and cytochrome $b$ (over $3100 \mathrm{bp}$ ) from eight individuals of Madeiran wall lizards, Lacerta dugesii, from four distinct island populations. The tRNAs exhibit a high degree of intraspecific polymorphisms compared to other vertebrates. All CR sequences include a minisatellite that varies in length between populations but is apparently fixed within them. Variation in minisatellite length appears between populations separated by apparently very short evolutionary time spans. Many motifs identified in the CR of other vertebrates are not highly conserved, although conserved blocks are identifiable between the few published reptile $\mathrm{CR}$ sequences. Overall there are extensive differences in the internal organization of the reptile CR compared to the more widely studied mammals and birds. Variability in the CR is lower than in cytochrome $b$, but higher than in 12S rRNA. Phylogenetic analysis of these sequences produces a well-resolved estimate of relationships between populations.
\end{abstract}

Key words: Lacerta dugesii - Control region D-Loop - Structure - Madeira - tRNA $^{\text {Thr }}-$ tRNA $^{\text {Pro }}-$ tRNA $^{\text {Phe }}$

\section{Introduction}

In vertebrates, the mitochondrial control region $(\mathrm{CR})$, is the major noncoding fragment of the molecule. The CR spans between the $t R N A^{\text {Phe }}$ and the $t R N A^{\text {Pro }}$ genes and includes the transcription initiation sites for both strands (Attardi 1985; Clayton 1982; Saccone et al. 1987). Because the CR lacks typical coding constraints, it accumulates base substitutions, indels, and a variable number of tandem repeats which are responsible for the extensive size variation found in the mtDNA molecule (Hoelzel et al. 1994; Sbisà et al. 1997). The CR is usually thought to be the fastest-evolving region of the genome (Brown et al. 1979), and for that reason it is broadly used to perform intra- and interspecific phylogenetic studies. However, only a few CR sequences have been published for reptiles (e.g., Sites et al. 1996; Kumazawa and Nishida 1999; Janke et al. 2001), so little is known concerning its comparative structure or phylogenetic utility within this group. Our aim was to address this by sequencing the CR for several individuals of the lacertid lizard Lacerta dugesii.

Lacerta dugesii is an endemic lizard from Madeira, the Desertas Islands, Porto Santo, and the Selvagens and was recently introduced to the Azores. Because of the well-known geological history of these islands (Geldmacher et al. 2000) and the high population densities of the lizards, it is a model organism for 


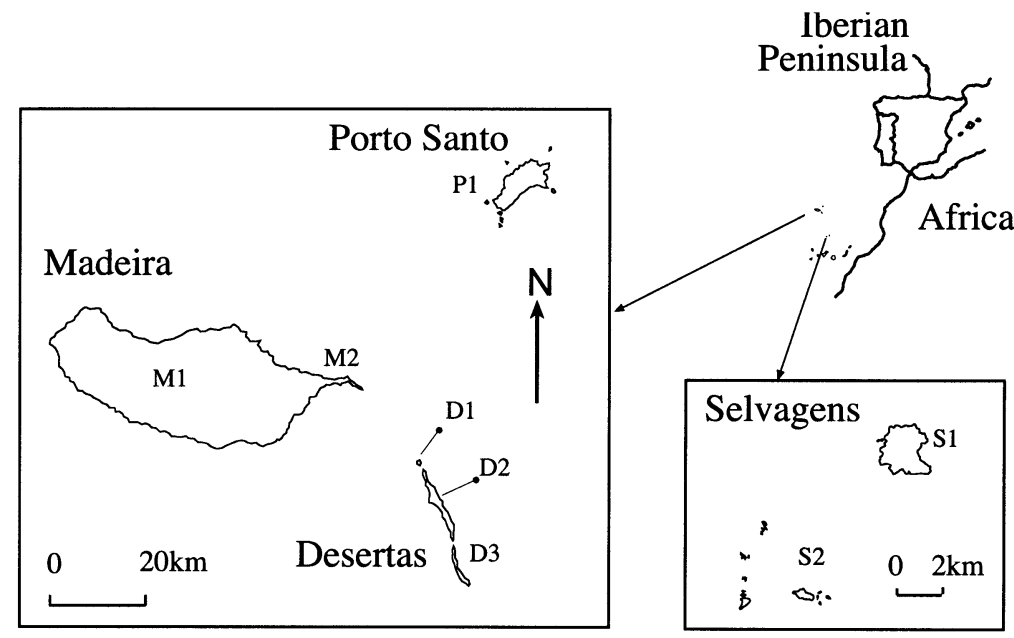

Fig. 1. Geographic location of the eight Lacerta dugesii specimens used in the present study. M, Madeira Island; D, Desertas Islands; P, Porto Santo Island; S, Selvagens. M1, Curral das Freiras; M2, Ponta de São Lourenço; D1, Bugio; D2, Deserta Grande; D3, Ilhéu Chão; S1, Selvagem Grande; S2, Selvagem Pequena. studying genetic variation across a fragmented habitat. Eight CR sequences from Lacerta dugesii individuals were compared interspecifically and with other published reptile sequences. We use this to address relative rates of evolution between CR, cytochrome $b$, and $12 \mathrm{~s}$ rRNA sequences. Within the CR we also investigate the evolutionary rate in putatively conserved regions and within the arrays of tandem repeats that commonly occur in both extremities of the CR (e.g., Savolainen et al. 2000).

\section{Materials and Methods}

\section{Laboratory Methods}

Eight specimens of Lacerta dugesii covering the species distribution range were sequenced for the entire CR (D-loop). The precise localities and code names of the specimens captured are shown in Fig. 1 and are as follows: two animals from Madeira Island (M1 and M2), three from the Desertas archipelago (D1, D2, and D3), one from Porto Santo Island (P1), and two from the Selvagens archipelago (S1 and S2). Total DNA was extracted from tail tips from each individual, followed by a phenol-chloroform standard procedure. The entire CR including three tRNAs and partial $12 \mathrm{~S}$ and cytochrome $b$ gene sequences were amplified in a single step using two primers, cBL (5'-CTGCATCTACCTCCACATCGGACG-3') and 12L (5'-AAGTTTTTCACTTGTAGTTCTCTG GCGG-3') and TaqPlus Long from Stratagene, following the manufacturer's instructions. PCR cycle conditions were $30 \mathrm{~s}$ at $94^{\circ} \mathrm{C}, 30 \mathrm{~s}$ at $60^{\circ} \mathrm{C}$, and $2 \mathrm{~min}$ at $70^{\circ} \mathrm{C}$, for 35 cycles. Primers cBL and $12 \mathrm{~L}$ were designed to anneal to conserved regions in the cytochrome $b$ and $12 \mathrm{~S}$ rRNA genes, respectively. Following the amplification of this fragment, nine primers were successively designed to amplify and sequence consecutive segments using the initial fragment as a template, until sequences from both extremities overlapped. Primer sequences and their relative locations and amplification conditions are described on the web page: www.uma.pt/bioarticles.

Amplified fragments were always sequenced three times and in both directions, on a 377 Applied Biosystem DNA Sequencing Apparatus, with the same set of primers used for amplification. One primer pair was specifically designed to amplify the region containing a repeat motif. We amplified this fragment in 10 individuals from each site and examined variation of the number of repeats within populations through differences in the length of
PCR products visualized in an agarose gel stained with ethidium bromide.

The existence of nuclear copies of mitochondrial sequences in a variety of organisms is well known [Numts according to Lopez et al. (1994)] and these may obscure inferences on mtDNA evolution (Bensasson et al. 2001; Woiscnik and Moraes 2002). The sequences initially appear to be mtDNA and not nuclear copies for several reasons. The strong bias against guanosines in the third positions of the cytochrome $b$ gene is typical in reptiles (Harris 2002). Also, part of the cytochrome $b$ and $12 \mathrm{~S}$ rRNA sequences overlap with previously published sequences of $L$. dugesii for these regions, and differ from them by less than $1 \%$ (Oliverio et al. 2000; Harris et al. 1998). Further, the free energy of the secondary structure of the 12S rRNA is similar to that of other lacertid species (data not shown; see Harris 2001), although this method does not always clearly identify numts (Olsen and Yoder 2002). To be completely sure that we had not amplified a nuclear copy we followed the same strategy as Bensch and Härlid (2000) and Saunders and Edwards (2000) and purified mitochondria from a fresh liver sample of $L$. dugesii in a $\mathrm{CsCl}$-ethidium bromide density gradient. A fragment was then amplified using the same set of primers and mtDNA extract as the template. Sequences obtained were identical to those obtained when using PCR products amplified from total DNA.

\section{Structural and Sequence Analysis}

The eight sequences were aligned using SEAVIEW (Galtier et al. 1996) and sequence similarities were checked by visual inspection. The sequences reported here are available in GenBank, accession numbers AY147872-AY147879. Analyses of codon positions and variable informative sites were done using PHYLO_WIN (Galtier et al. 1996). Search for possible sequence motifs repetitions in the $\mathrm{CR}$ and comparison with other taxa were done using BLASTN v.2.2.1 (Altschul et al. 1997). The MFOLD program (Zuker et al. 1999) was employed to identify the three tRNAs' secondary structures. The search for potential promoter sequences was done with PROSCAN v1.7 and NNPP, available at the BCM web site. We have searched for potential secondary structures around conserved segments or segments identified as highly conserved across several organisms using MFOLD and RNAdraw (Matzura and Wennborg 1996). Sequences were phylogenetically analyzed using PAUP* 4.0.b3a (Swofford 2001).

When estimating phylogenetic relationships among sequences, one assumes a model of evolution regardless of the optimality criteria employed. Here we used the approach suggested by Huelsenbeck and Crandall (1997) to test 56 alternative models of evo- 


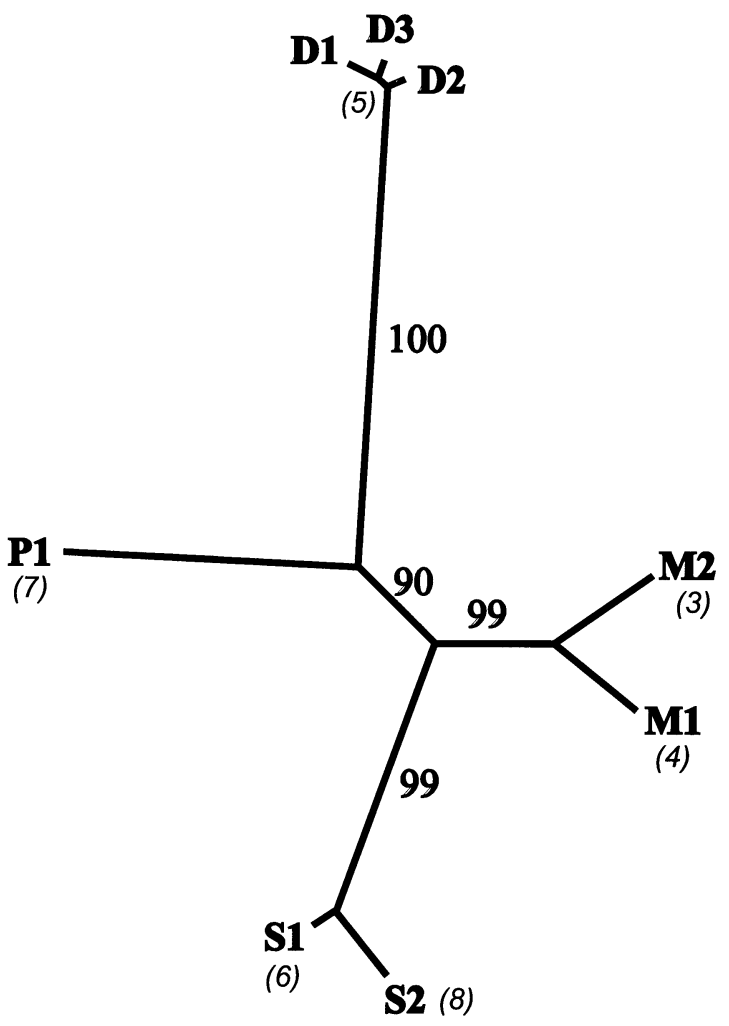

\subsection{5 substitutions/site}

Fig. 2. Phylogenetic tree based on the complete sequences of eight Lacerta dugesii specimens. Abbreviations are as in Fig. 1. Numbers on branches are bootstrap values (see text). In parentheses are the numbers of $37-b p$ repeats in the control region.

lution, using MODELTEST v.3 (Posada and Crandall 1998), described in detail by Harris and Crandall (2000). The chosen model of evolution was used to estimate a tree using maximum likelihood (Felsenstein 1981), with confidence in resulting nodes assessed using the bootstrap technique (Felsenstein 1985) with 1000 pseudoreplicates.

\section{Phylogenetic Analysis}

The three major regions sequenced (12S rRNA, cytochrome $b$, and $\mathrm{CR})$ were analyzed separately and combined. For the combined analysis the most appropriate model of evolution was the TVM, with an estimated proportion of invariable sites (0.1) and a discrete approximation of the $\Gamma$ distribution (0.001). A heuristic search with 100 replicates with this model found a single most likely tree of $-\ln$ 6385 (Fig. 2).

\section{Results}

\section{Structure of the Amplified Region}

The CR and adjacent gene sequences were obtained from eight Lacerta dugesii individuals. The aligned sequences varied in length from 3112 to $3311 \mathrm{bp}$ and included $851 \mathrm{bp}$ of the cytochrome $b$ gene, the complete $t R N A^{T h r}\left(69 \mathrm{bp}\right.$ ) and $t R N A^{\text {Pro }}$ genes (varying between 69 and $70 \mathrm{bp}$ ), the whole CR, the complete $t R N A^{\text {Phe }}$ gene (which varied in length from $75 \mathrm{bp}$ in five individuals to $74 \mathrm{bp}$ in three others), and a partial sequence from the $12 \mathrm{~S}$ rRNA gene which varied from 478 bp (in D1, D2, and D3) to 480 bp (M1, M2, S1, and $\mathrm{S} 2)$ to $481 \mathrm{bp}(\mathrm{P} 1) . t R N A^{T h r}$ is separated from the cytochrome $b$ gene by a sequence of six nucleotides. Table 1 lists the nucleotide frequencies of the cytochrome $b$ gene, the CR, the $12 \mathrm{~S}$ rRNA gene, and the three tRNAs together. The transition/transversion ratio $\left(T_{\mathrm{s}} / T_{\mathrm{v}}\right)$ of the cyt $b$ sequence is 9.5 , with a clear bias toward $\mathrm{C} \leftrightarrow \mathrm{T}(\mathrm{AG} / \mathrm{CT}=0.44)$, most of the substitutions being at the third codon position. The same applies to the $12 \mathrm{~S}$ rRNA sequence $\left(T_{\mathrm{s}} / T_{\mathrm{v}}=\right.$ 3.43; AG/CT $=0.85$ ). Table 1 compares the number of variable sites of the four regions surveyed with those that are phylogenetically informative.

\section{Transfer RNA Genes}

The base content of the three tRNAs of L. dugesii is similar to values found in other organisms (Table 1). The $T_{\mathrm{s}} / T_{\mathrm{v}}$ ratio observed in the three tRNAs is 12 : only 1 transversion found, versus 12 transitions (AG/ $\mathrm{CT}=1)$. Lacerta dugesii tRNAs appear to be functional, encoding apparently stable secondary struc-

Table 1. Number of sites found in Cyt $b$, the CR, the repeat region in the $5^{\prime}$ left CR strand (CR minisatellite), 12S RNA, and three tRNAs ${ }^{\mathrm{a}}$

\begin{tabular}{|c|c|c|c|c|c|c|c|c|}
\hline Region & Codon position & NS & $\% \mathrm{~A}$ & $\% \mathrm{C}$ & $\% \mathrm{G}$ & $\% \mathrm{~T}$ & NVS (\%) & NIS (\%) \\
\hline \multirow[t]{4}{*}{ Cyt $b$} & 1 & 283 & 25.0 & 30.2 & 22.4 & 22.3 & $16(5.7)$ & $8(2.8)$ \\
\hline & 2 & 284 & 20.3 & 24.0 & 12.1 & 43.6 & $5(1.8)$ & $3(1.1)$ \\
\hline & 3 & 284 & 33.7 & 41.4 & 4.3 & 20.7 & 85 (29.9) & $61(21.5)$ \\
\hline & Total & 851 & 26.4 & 31.9 & 12.9 & 28.9 & $106(12.5)$ & $72(8.5)$ \\
\hline & Total & 1408 & 32.8 & 22.1 & 9.7 & 35.5 & $97(6.9)$ & $67(4.8)$ \\
\hline \multirow{2}{*}{$\mathrm{CR}$ minisatellite } & & $36^{\mathrm{b}}$ & 12.5 & 42.5 & 16.7 & 28.1 & & \\
\hline & & $39^{\mathrm{c}}$ & 19.5 & 44.0 & 15.9 & 28.1 & & \\
\hline $12 \mathrm{~S}$ RNA & Total & 478 & 34.4 & 23.0 & 18.4 & 24.2 & $31(6.5)$ & $22(4.6)$ \\
\hline tRNAs & Total & 212 & 33.4 & 26.1 & 18.1 & 22.3 & $13(6.1)$ & $6(2.8)$ \\
\hline
\end{tabular}

\footnotetext{
${ }^{a}$ Nucleotide frequencies in the four regions are given, as well as the number of variable sites (NVS), and, of these, the number that are phylogenetically informative (NIS). CR values do not include the repeats. NS, number of sites.

${ }^{b}$ Number of sites in each repeat without gaps.

${ }^{\mathrm{c}}$ Number of sites in each repeat with gaps.
} 
a)

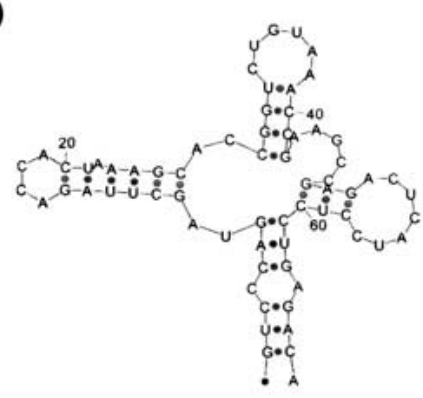

b)
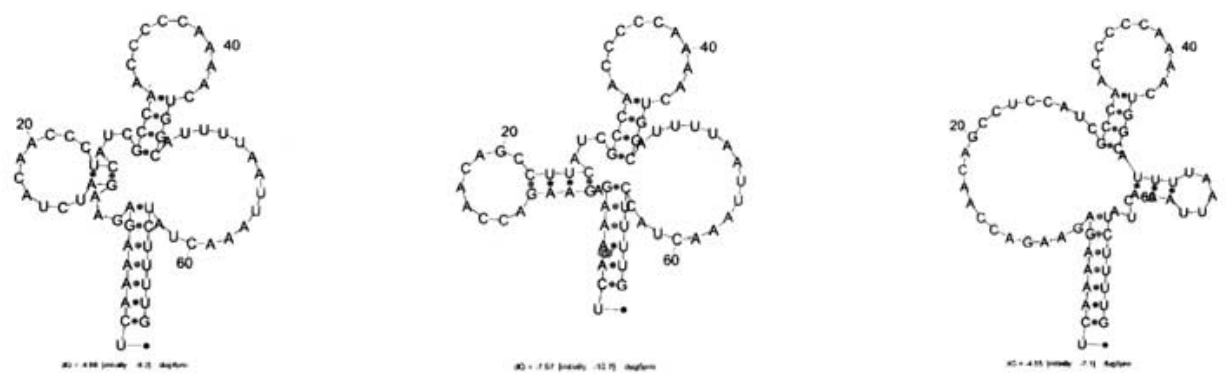

c)
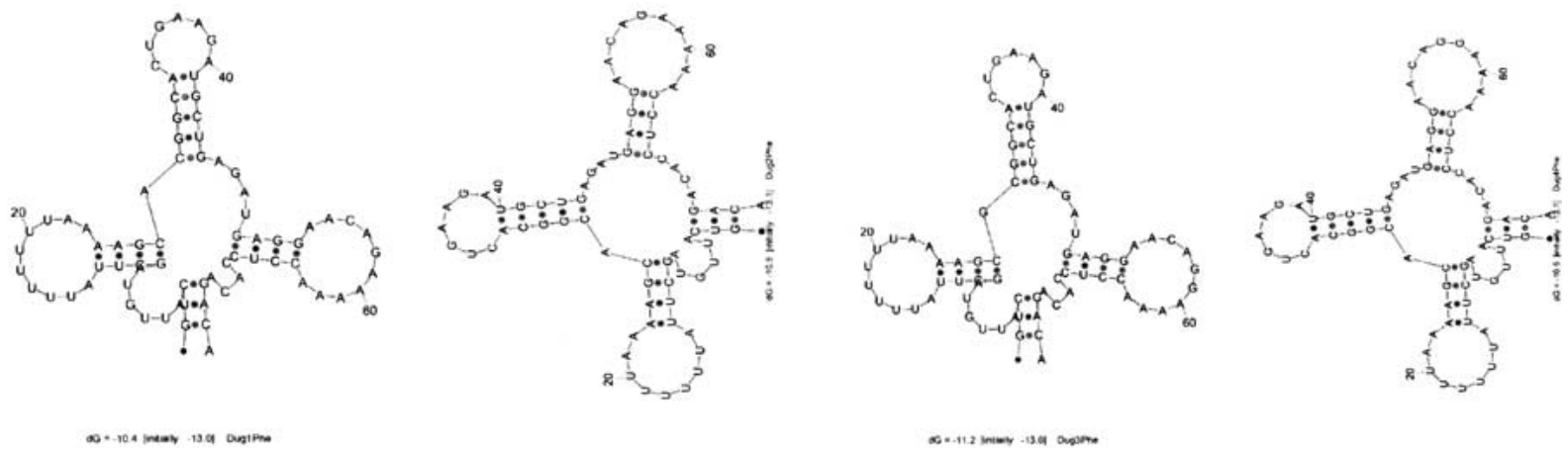

Fig. 3. Secondary structures of the presumably functional $t R N A^{T h r}(\mathbf{a}), t R N A^{\text {Pro }}$ (b), and $t R N A^{\text {Phe }}$ (c) genes.

tures usually present in mitochondrial tRNAs $(\mathrm{Ku}-$ mazawa and Nishida 1993). Due to an intraspecific polymorphism the $t R N A^{T h r}$ gene may assume two different stable secondary structures (Fig. 3a) with perfect cloverleaf topologies. The $t R N A^{\text {Pro }}$ gene is much more variable (Fig. 3b). The eight $L$. dugesii individuals gave six $t R N A^{\text {Pro }}$ sequences (Fig. 3c). This is not an unusual feature of this tRNA (Kumazawa and Nishida 1995), but the extent of intraspecific polymorphism in this species is extremely high. The fact that no duplicate forms of the $t R N A^{\text {Pro }}$ gene were found allows us to assume that all are functional structures, although it easily accumulates indels or base substitutions.

\section{The Control Region (CR)}

The Lacerta dugesii $\mathrm{CR}$ is depicted schematically in Fig. 4. The CR base composition is characterized by a low $\mathrm{G}$ content $(9.7 \%)$, as is typical in vertebrates. The base content $\mathrm{GC}$ versus AT is irregular across the entire region and we did not attempt to divide the 


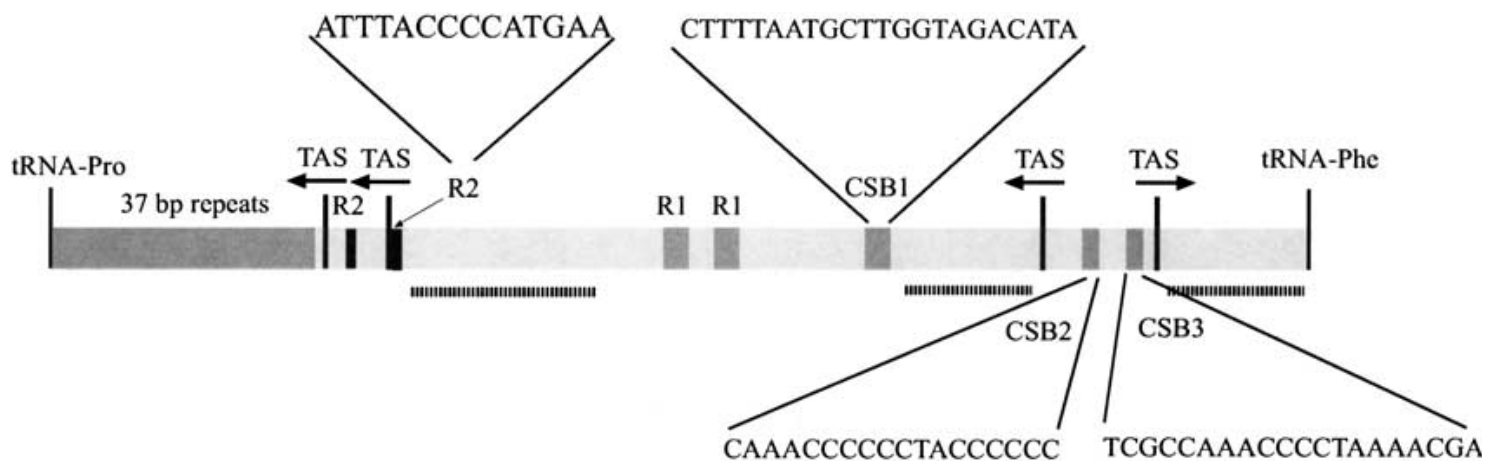

Fig. 4. General structure of the control region in Lacerta dugesii. R1 and R2 are 31- and 14-bp perfect repeats. The dark gray region adjacent to $t R N A^{\text {Pro }}$ represents the variable number of tandem 37-bp repeats. The dotted bars below the gray bar show the localizations of highly variable $C R$ regions.

segment into domains because it is not comparable between different species. The overall $T_{\mathrm{s}} / T_{\mathrm{v}}$ ratio in the $\mathrm{CR}$ is 3.43 (21 transversions and 72 transitions; $\mathrm{AG} / \mathrm{CT}=0.5)$. Figure 4 illustrates the distribution of repeat and variable sites as well as three CSB (conserved sequence blocks) and TAS (termination-associated sequence) elements. The Lacerta dugesii CR structure does not present the typical domains described for mammals (Randi and Lucchini 1998). The CR presents two well-conserved central domains (about $130 \mathrm{bp}$ each) with no variable sites, flanked by variable domains. The sequence between the two central conserved segments (about $260 \mathrm{bp}$ ) is also variable. The extreme left domain (the $5^{\prime}$ terminus of the nascent $\mathrm{H}$-strand) contains tandem repeats consisting of four to nine 37-bp repeats responsible for the variable length observed in the mtDNA CR. The base composition of this minisatellite is very different from that of the total CR (Table 1), with a striking increase in cytosines and deficit of adenines. The number of these repeats was found to be constant in 10 individuals screened from each population. Figure 4 also illustrates how conserved blocks are interspaced with more variable sections. Two repeats of 34 bp were found in a conserved region, separated by 28 bp (R1 in Fig. 4). Four motifs matching the TAS element $\left(5^{\prime}\right.$...AATTACA.... $\left.3^{\prime}\right)$ reported by Saccone et al. (1987), as well as the more expanded sequence denominated ETAS (Sbisà et al. 1997), are evident (Fig. 4), but three of them are in an inverted direction from that of Saccone et al. (1987). The conservative nature of these sequences and the flanking regions provides additional evidence that they may be of importance for mitochondrial transcription and regulation in the D-loop-containing region (Brown et al. 1986; Saccone et al. 1987; Sbisà et al. 1997). The four TAS elements do not seem to be randomly distributed in the CR since they are found in the extremities and in conserved regions. Brown et al. (1986) reported the existence of four TAS motifs in rodent, cow, and human mtDNAs, all located in the $3^{\prime}$ end of the D-loop region. Three CSB elements (CSB-1, -2, and -3 in Fig. 4) are highly conserved among the eight L. dugesii specimens. TATATA repeats, the most common motif for transcription by RNA polymerase II, are randomly dispersed across the whole CR. Also, the motif CCAATC (probably with a function identical to that of the TATA boxes) was found in the conserved segment between R1 and CSB1 and also near the $3^{\prime}$ end, next to $t R N A^{\text {Phe }}$. A common modular element for most promoters, the ATATAA box, is also present between the $\mathrm{R} 2$ repeats. Three GYRCAT motifs $(\mathrm{Y}=\mathrm{C} / \mathrm{T} ; \mathrm{R}=\mathrm{A} / \mathrm{G})$ are evident, two of them included in the already-mentioned TAS boxes. These motifs are quite widespread in Domain I of mammals and birds (Randi and Lucchini 1998), but they are also part of the more general TAS (also called ETAS) motifs. We have searched for possible open reading frames (ORFs) but no potential promoters were recognized in the CR with the search tools used. Two TCCC motifs exist in the Lacerta dugesii CR, which have been linked to termination of $\mathrm{H}$ strands in mammalian and bird D-loops (Douzery and Randi 1997; Randi and Lucchini 1998) but neither are linked to putative cloverleaf secondary structures.

\section{Phylogenetic Value of the $C R$}

Our analysis of the combined sequences produces a single well-resolved estimate of phylogeny. Individuals from each of the island groups are strongly supported as clades - three individuals from the Desertas (100\% bootstrap support), two from Madeira (99\%), and two from the Selvagens (100\%). The individuals from the Selvagens and Madeira form a clade relative to those from the Desertas and Porto Santo (90\%) (Fig. 2). In the separate analysis of $12 \mathrm{~S}$ rRNA, cytochrome $b$, and CR sequences, all of these clades are similarly recovered (data not shown). 


\section{Discussion}

\section{Structure and Comparative Analysis of the CR}

We did not attempt to divide the Lacerta dugesii CR into domains according to degree of variability or base content, as this division would be arbitrary in this species. The Lacerta dugesii $\mathrm{CR}$ shows a strong bias against guanine on the light strand $(\mathrm{G}=9.7 \%)$, slightly lower than the values found in other reptiles (Macey et al. 1997a). The $t R N A^{\text {Pro }}$ gene appears to be under relaxed selection to accumulate a considerable number of indels and base substitutions, even within a species. The presence of tandem repeats near the $3^{\prime}$ end of the CR is not uncommon in vertebrates, although their putative function and origin are unknown. Repeats of variable number and length have been found in various organisms, but not always localized in the same domains (Moritz and Brown 1986; Lunt et al. 1998; Savolainen et al. 2000): turtles (Zardoya and Meyer 1998; Kumazawa and Nishida 1999), bears (Matsuhashi et al. 1999), snakes and skinks (Kumazawa et al. 1998; Kumazawa and Nishida 1999), and iguanas (Janke et al. 2001). The several types of repeats found in L. dugesii do not follow the general pattern described by Hoelzel et al. (1994) and Savolainen et al. (2000) for vertebrates in general. It is interesting to observe that the sequence CAACAAA also appears twice in $L$. dugesii, once in the middle and in the left domains of the CR light strand. In Iguana iguana, the same sequence appears three times consecutively (as in gulls), in the left domain of the CR, immediately before the CSB-2 sequence. Macey et al. (1997b) have found repeats between tandemly duplicated pairs of the $t R N A^{\text {Pro }}$ gene of several species. It is thus plausible that the $5^{\prime}$ TTTTG3 $3^{\prime}$ present in each repeat following the $L$. dugesii $t R N A^{\text {Pro }}$ may in fact be reminiscent of imperfect duplications of this tRNA. TAS elements have been associated with stop sites for DNA synthesis (Doda et al. 1981) and have been found near the $3^{\prime}$ terminus of the D-loop strand. The ones reported in this work perfectly match those reported in snakes [in both sequence and direction (Kumazawa et al. 1998)] and are identical, but inverted, to the ones reported by Saccone et al. (1987) for vertebrates in general. The Lacerta dugesii CR has two TAS sequences in both the $3^{\prime}$ and the $5^{\prime} \mathrm{CR}$ extremities, contrary to their clustering only in the $3^{\prime}$ extremity of rodents (Brown et al. 1986) and Galliformes (Randi and Lucchini 1988 and references therein). Another TAS motif is the sequence GYRCAT, which was found to be highly conserved in birds as well as mammals. The same sequence exists in $L$. dugesii in the CR left region, first as GTACAT and then as the more common GTGCAT. The segment can form a structure with four hairpins and loops with $\Delta G=$
$-23 \mathrm{kcal}$. In I. iguana this motif is repeated five times, three of them in the first 215 CR bases.

Another common feature associated with the CR of all organisms reported so far is the existence of CSBs associated with start sites for DNA synthesis. In the eight sequences of Lacerta dugesii the three CSB blocks are found in conserved regions (Fig. 4). CBS-2 has a motif identical in L. dugesii, I. iguana (Janke et al. 2001), and Eumeces egregious (Kumazawa and Nishida 1999). Interestingly, while similarities between reptile and mammal CSB-2 are high, we could not identify correlated sequences from any of the CR sequences of birds. CBS-3 is also quite similar across reptiles, with only a few differences in the I. iguana sequence. Again, there is evident homology among reptiles, mammals, and birds. The least conserved block among the published reptile species is CSB-1, although the sequences are still quite similar. It is evident that among these reptile species, CSB boxes are highly conserved, supporting the view that the asymmetrical replication mechanisms proposed for mammalian mtDNAs are the same for reptiles (Kumazawa and Nishida 1999). Although some motif sequences can be found in all vertebrates included in the comparison, it is hard to find CSB-1 homologies among mammals, birds, and reptiles. This is not surprising since Crochet and Desmarais (2000) had already reported strong differences in this sequence and related secondary structure within gulls. It seems that CSB-1 is not so highly conserved across vertebrates. In $L$. dugesii the CSB-1 block and the preceding $100 \mathrm{bp}$ are nonvariable and are able to form a stable secondary structure. It has been hypothesized that conserved sequences like CSBs or conserved CR domains can form stable secondary structures (Brown et al. 1986; Crochet and Desmarais 2000). However, except for CBS-1, the other two CSBs do not form stable secondary structures. We failed to identify a sequence that encompasses $\mathrm{H}$-strand initiation in $L$. dugesii or the transcriptional promoter, unless this sequence is the $\operatorname{poly}(\mathrm{C})$ of the CSB-2 motif, just a few bases downstream from a TATA box. The CSB-1 motif also presents a conserved AGACAT motif, similar to the putative GGACAT apparently linked to mitochondrial LSP/HSP (Randi and Lucchini 1998). The presence of two STOP signals following these motifs could be used to abort transcription of the H-strand. In this regard $L$. $d u$ gesii CR organization finds parallels in mammals and birds (Clayton 1982; Sbisà et al. 1997; Randi and Lucchini 1998). Overall, however, we observe strong differences in the internal organization of the reptile CR compared with the mammals and birds studied so far. It should be interesting to screen the CR of more reptile families to see if the extent of variation is a particular feature of this group of vertebrates, which would have important evolutionary implications. 


\section{Evolutionary Implications of the L. dugesii $C R$}

From our estimate of phylogenetic relationships it is clear that the number of repeat sequences has changed multiple times in different lineages. The two populations in Madeira differ (three or four repeats), as do the populations in the Selvagens (six or eight repeats). These populations are strongly supported as single lineages (100\% bootstrap support), indicating that changes in repeat number occurred after these clades separated from the other populations. To obtain a rough estimate of timing of cladogenic events within the phylogeny, we used a likelihood ratio test to assess whether the sequences were evolving in a clocklike fashion. We included only the $12 \mathrm{~S}$ rRNA and cytochrome $b$ sequences, since there is a wellcalibrated estimate of $1.96 \%$ sequence divergence per million years for lacertid lizards, Gallotia, from the Canary Islands for these regions (Carranza et al. 2000). The tree constructed under a molecular clock assumption is not significantly different from the ML tree (analysis not shown). Therefore we can estimate that the populations within the Desertas and within the Selvagens separated from each other about 100,000 years ago. The two Madeiran populations diverged approximately 900,000 years ago. From this we deduce that changes in repeat numbers, and their apparent fixation in populations, can occur in a short evolutionary time scale.

The CR has not been used much in phylogenetic studies in reptiles, probably because of the presence of repeat units and the lack of universal primers. Our study suggests that it can be highly informative for resolving intraspecific phylogenies. Moreover, our identification of conserved structures between divergent reptile groups should facilitate primer design within the CR. For closely related taxa variation in the number of repeats in the CR could also be a phylogenetically useful character.

Acknowledgments. The authors are grateful to Parque Natural da Madeira for providing some of the specimens used in the study. We acknowledge Professor António Amorim of IPATIMUP (Porto, Portugal) for support and the facilities provided. Thanks are also due to Dr. Ana Tenreiro, Sciences Faculty of Lisbon University, for the isolation of mitochondria. This investigation was partially funded by Grant PBIC/P/CEN/1096/92 from Fundação da Ciência Tecnologia (Lisbon) to L.V.

\section{References}

Altschul SF, Madden TL, Schäffer AA, Zhang J, Zhang Z, Miller W, Lipman D (1997) Gapped BLAST and PSI-BLAST: A new generation of protein database search programs. Nucleic Acids Res 25:3389-3402

Attardi G (1985) Animal mitochondrial DNA: An extreme example of genetic economy. Int Rev Cytol 93:93-148
Bensasson D, Zhang DX, Hartl D, Hewitt GM (2001) Mitochondrial pseudogenes: Evolution's misplaced witnesses. TEE 16 (http://tree.trends.com 0169-5347/01/\$)

Bensch A, Härlid A (2000) Mitochondrial genomic rearrangements in songbirds. Mol Biol Evol 17:107-113

Brown G, Gadaleta G, Pepe G, Saccone C, Sbisà E (1986) Structural conservation and variation in the D-loop-containing region of vertebrate mitochondrial DNA. J Mol Biol 192:503-511

Brown WM, George M Jr, Wilson AC (1979) Rapid evolution of animal mitochondrial DNA. Proc Natl Acad Sci USA 76:19671971

Carranza S, Arnold EN, Mateo JA, Lopez-Jurado LF (2000) Long-distance colonization and radiation in gekkonid lizards, Tarentola (Reptilia: Gekkonidae), revealed by mitochondrial DNA sequences. Proc R Soc Lond B 267:637-649

Clayton DA (1982) Replication of animal mitochondrial DNA. Cell 28:693-705

Crochet PA, Desmarais E (2000) Slow rate of evolution in the mitochondrial control region of gulls (Aves: Laridae). Mol Biol Evol 17:1797-1806

Doda DA, Wright CT, Clayton DA (1981) Elongation of displacement-loop strands in human and mouse mitochondrial DNA is arrested near specific template sequences. Proc Natl Acad Sci USA 78:6116-6120

Douzery E, Randi E (1997) The mitochondrial control region of Cervidae: Evolutionary patterns and phylogenetic contents. Mol Biol Evol 14:1154-1166

Felsenstein J (1981) Evolutionary trees from DNA sequences: A maximum likelihopd approach. J Mol Evol 17:368-376

Felsenstein J (1985) Confidence limits on phylogenies: An approach using the bootstrap. EvoIution 39:783-791

Galtier N, Gouy M, Gautier C (1996) SEAVIEW and PHYLO_WIN: Two graphic tools for sequence alignment and molecular phylogeny. Comput Appl Biosci 12:543-548

Geldmacher J, Bogaard P, Hoernle K, Schnincke HU (2000) The ${ }^{40} \mathrm{Ar}^{39}$ age dating of the Madeira Archipelago and hotspot track (eastern North Atlantic). Geochem Geophys Geosyst 1: http://g-cubed.org

Harris DJ (2001) Re-evaluation of 16S ribosomal RNA variation in Bufo (Anura: Amphibia). Mol Phylogenet Evol 19:326-329

Harris DJ (2002) Reassessment of comparative genetic distance in reptiles from the mitochondrial cytochrome $b$ gene. Herpetol J (in press)

Harris DJ, Crandall KA (2000) Intragenomic variation within ITS1 and ITS2 of freshwater crayfishes (Decapoda: Cambaridae): Implications for phylogenetic and microsatelitte studies. Mol Biol Evol 17:284-291

Harris DJ, Arnold EN, Thomas RH (1998) Relationships of lacertid lizards (Reptilia: lacertidae) estimated from mitochondrial DNA sequences and morphology. Proc R Soc Lond B 265:1939-1948

Hoelzel AR, Lopez JV, Dover GA, O’Brien SJ (1994) Rapid evolution of a heteroplasmic repetitive sequence in the mitochondrial DNA control region of carnivores. J Mol Evol 39:191-199

Hulsenbeck JP, Crandall KA (1997) Phylogenetic estimation and hypothesis testing using maximum likelihood. Annu Rev Ecol Syst 28:437-466

Janke A, Erpenbeck D, Nilsson M, Arnason U (2001) The mitochondrial genomes of the iguana (Iguana iguana) and the caiman (Caiman crocodylus): Implications for amniote phylogeny. Proc R Soc Lond B Biol Sci 268:623-631

Kumazawa Y, Nishida M (1993) Sequence evolution of mitochondrial tRNA genes and deep-branch animal phylogenetics. J Mol Evol 37:380-398 
Kumazawa Y, Nishida M (1995) Variations in mitochondrial tRNA gene organization of reptiles as phylogenetic markers. Mol Biol Evol 12:759-772

Kumazawa Y, Nishida M (1999) Complete mitochondrial DNA sequences of the green turtle and blue-tailed skink: Statistical evidence for archosaurian affinity of turtles. Mol Biol Evol 16:784-792

Kumazawa Y, Ota H, Nishida M, Ozawa T (1998) The complete nucleotide sequence of a snake (Dinodon semicarinatus) mitochondrial genome with two identical control regions. Genetics 150:313-329

Lopez JV, Yuhki N, Masuda R, Modi W, O'Brien SJ (1994) Numt, a recent transfer and tandem amplification of mitochondrial DNA to the nuclear genome of the domestic cat. J Mol Evol 39:174-190

Lunt DH, Whipple LE, Hyman BC (1998) Mitochondrial DNA variable number of tandem repeats (VNTRs): utility and problems in molecular ecology. Mol Ecol 7:1441-1455

Macey JR, Larson A, Ananjeva NB, Fang Z, Papenfuss T (1997a) Two novel gene orders and the role of light-strand replication in rearrangement of the vertebrate mitochondrial genome. Mol Biol Evol 14:91-104

Macey JR, Larson A, Ananjeva NB, Papenfuss T (1997b) Replication slippage may cause parallel evolution in the secondary structures of mitochondrial transfer RNAs. Mol Bio Evol 14:30-39

Matsuhashi T, Masuda R, Mano T, Yoshida MC (1999) Microevolution of the mitochondrial DNA control region in the Japanese brown bear (Ursus arctos) population. Mol Biol Evol 16:676-684

Matzura O, Wennborg A (1996) RNAdraw: an integrated program for RNA secondary structure calculation and analysis under 32-bit Microsoft Windows. Comput Appl Biosci $12: 247-249$

Moritz C, Brown WM (1986) Tandem duplications of D-loop and ribosomal RNA sequences in lizard mitochondrial DNA. Science 233:1425-1427

Oliverio M, Bologna MA, Mariottini P (2001) Molecular biogeography of the Mediterranean genera Podarcis and Teira (Reptilia, Lacertidae). J Biogeogr 27:1403-1420
Olson LE, Yoder AD (2002) Using secondary structure to identify ribosomal Numts: Cautionary examples from the human genome. Mol Biol Evol 19:93-100

Posada D, Crandall KA (1998) MODELTEST: Testing the model of DNA substitution. Bioinfomatics 14:817-818

Randi E, Lucchini V (1998) Organization and evolution of the mitochondrial DNA control region in the avian genus Alectoris. J Mol Evol 47:449-462

Saccone C, Attimonelli MA, Sbisà E (1987) Structural elements highly preserved during the evolution of the D-Loop containing region in vertebrate mitochondrial DNA. J Mol Evol 26:205-211

Saunders MA, Edwards SV (2000) Dynamics and phylogenetic implications of mtDNA control region sequences in New World jays (Aves: Corvidae). J Mol Evol 51:97-109

Savolainen P, Arvestad L, Lundeberg J (2000) mtDNA tandem repeats in domestic dogs and wolves: Mutation mechanism studied by analysis of the sequence of imperfect repeats. Mol Biol Evol 17:474-488

Sbisà E, Tanzariello F, Reyes A, Pesole G, Saccone C (1997) Mammalian mitochondrial D-loop region structural analysis: Identification of new conserved sequences and their functional and evolutionary implications. Gene 205:125-140

Sites JL Jr, Davis S, Guerra T, Iverson J, Snell H (1996) Character congruence and phylogenetic signal in molecular and morphological data sets: A case study in the living iguanas (Squamata, Iguanidae). Mol Biol Evol 13:1087-1105

Swofford DL (2001) PAUP*4.0.b3a: Phylogenetic analysis using parsimony (and other methods). Sinauer Associates, Sunderland, MA

Woischnik M, Moraes CT (2002) Pattern of organization of human mitochondrial pseudogenes in the nuclear genome. Genome Res 12:885-893

Zardoya R, Meyer A (1998) Complete mitochondrial genome suggests diapsid affinities of turtles. Proc Nat Acad Sci USA 95:14226-14231

Zuker M, Mathews DH, Turner DH (1999) Algorithms and thermodynamics for RNA secondary structure prediction: A practical guide. In: Barciszewski J, Clark BFC (eds) RNA biochemistry and biotechnology NATO ASI Series. Kluwer Academic, Dordrecht, pp 11-43 